\title{
A rat mammary gland cancer cell with stem cell properties of self-renewal and multi-lineage differentiation
}

\author{
Cinzia Cocola $\cdot$ Sveva Sanzone $\cdot$ Simonetta Astigiano $\cdot$ Paride Pelucchi · \\ Eleonora Piscitelli · Laura Vilardo · Ottavia Barbieri · Gloria Bertoli · \\ Rolland A. Reinbold · Ileana Zucchi
}

Received: 11 September 2008/Accepted: 12 September 2008/Published online: 25 November 2008

(C) Springer Science+Business Media B.V. 2008

\begin{abstract}
The cancer stem cell hypothesis posits that tumors are derived from a single cancer-initiating cell with stem cell properties. The task of identifying and characterizing cancer-initiating cells with stem cell properties at the single cell level has proven technically difficult because of the scarcity of the cancer stem cells in the tissue of origin and the lack of specific markers for cancer stem cells. Here we show that a single LA7 cell, derived from rat mammary adenocarcinoma has: the ability to serially re-generate mammospheres in long-term non-adherent cultures, the differentiation potential to generate all the cell lineages of the mammary gland and branched duct-like structures that recapitulate morphologically and functionally the ductal-alveolar-like architecture of the mammary tree. The properties of
\end{abstract}

Cinzia Cocola, Sveva Sanzone and Simonetta Astigiano have contributed equally to this work.

C. Cocola $\cdot$ S. Sanzone $\cdot$ P. Pelucchi $\cdot$ E. Piscitelli .

L. Vilardo · G. Bertoli · I. Zucchi $(\bowtie)$

ITB-CNR, Via Cervi 93, 20090 Segrate (Milan), Italy

e-mail: ileana.zucchi@itb.mi.cnr.it

S. Astigiano - O. Barbieri

IST and DIMES, Università di Genova, L.go R. Benzi 10, 16132 Genova, Italy

R. A. Reinbold

Max Planck Institute, 48149 Muenster, Germany self-renewal, extensive capacity for proliferation, multi-lineage differentiation and the tubular-like structure formation potential suggest that LA7 cells is a cancer stem model system to study the dynamics of tumor formation at the single cell level.

Keywords LA7 cell line - Self-renewal · Cancer stem cells · Mammary gland multi-lineage differentiation - Three-dimensional differentiation $\cdot$ Epithelial markers

\section{Introduction}

Experimental evidence and clinical data suggest that adult stem cells may be the targets of mutations during adulthood (Bonnet and Dick 1997; Miyamoto et al. 2000; George et al. 2001; Reya et al. 2001). Tumors derived from cancer stem cells contain a hierarchical population of cells in which only a small number of cells possess tumor initiating potential ("tumor initiating cell") and the majority are differentiated cells. This indicates that tumor stem cells retain the normal stem cells properties of selfrenewing and differentiation potential.

The existence of adult stem cells in the mammary gland was demonstrated by showing that a functional mammary gland could be generated in immunodeficient mice by the transplantation of a single cell with stem cell properties (Shackleton et al. 2006; Stingl 
et al. 2006). The hypothesis that breast cancers arise from deregulation of stem cells is supported by the evidence that mammary tumors are frequently found to be clonal in origin (Going et al. 2001). The existence of mammary cancer stem cells was recently demonstrated by showing that tumor-initiating cells, enriched for $\mathrm{CD} 44^{+} \mathrm{CD} 24^{\text {low }}$ marker expression, when injected into NOD/scid mice, form morphologically heterogeneous tumors, similar to the tumors from which they were derived (Al-Hajj et al. 2003; Al-Hajj and Clarke 2004). Long-term non-adherent cultures represent the most suitable method for growing and enriching for breast tumor-initiating cells (Ponti et al. 2005). These cells, expanded in vitro in the form of mammospheres, expressed the cell surface antigen, CD44.

In the field of cancer stem cell biology, quantitative assays for single-cell isolation and analysis are essential to fully understand the tumorigenic properties and potential of cancer stem cells in solid tumors. The comprehensive characterization of the tumorigenic properties of cells with single-cell tumor formation potential depends on identifying and isolating single cancer-initiating cells. The task of identifying and characterizing a single cancer-initiating cell with stem cell properties has proven to be technically difficult because of the lack of universal and specific markers for cancer and somatic stem cells. In addition, cell lines have been of limited benefit in quantitative assays because cell lines usually do not recapitulate all aspects of the primary tumor.

In our laboratory we are using a mammary gland cancer stem cell model, based on LA7, polygonal cells that have the capacity to recapitulate certain aspects of mammary alveologenesis. The model was originated by Renato Dulbecco from an adenocarcinoma induced in rat (Sprague Dawley) by chemicals (Bennett et al. 1978; Dulbecco et al. 1979). In this study our aim was to investigate whether a single LA7 cell had the ability to generate and propagate as mammospheres in long-term nonadherent cultures, differentiate into all the cell lineages of the mammary gland and generate branched duct-like structures that recapitulate morphologically and functionally the architecture of the mammary tree.

The property of self-renewal, extensive capacity for proliferation, multi-lineage differentiation potential and the ability to develop tubular-like structures in 3D cultures, strongly support that the LA7 cells are cancer stem cells (Zucchi et al. 2007). We propose that LA7 cells can be used both as a stem cell model system to study the dynamics of breast tumor genesis and to study certain aspects of normal mammary development.

\section{Material and methods}

\section{Cell lines}

The cell line LA7 is a clonal derivative of the RAMA25 line, derived from a mammary adenocarcinoma induced in a rat with DMBA (dimethylbenzanthracene). Cells were cultured in DMEM, $10 \%$ fetal bovine serum (FBS, Sigma), supplemented with $50 \mathrm{ng} / \mathrm{mL}$ insulin (Sigma) and $50 \mathrm{ng} / \mathrm{mL}$ hydrocortisone (Sigma).

\section{Monolayer differentiation}

For induction of differentiation, confluent LA7 cells were treated with $2 \%$ DMSO (Sigma) for $60 \mathrm{~h}$. LA7 cells were harvested with $0.05 \%$ trypsin $-0.53 \mathrm{mM}$ EDTA (Invitrogen).

Mammospheres generation

LA7 cells $(1000 / \mathrm{mL})$ were plated in low attachment plates (Bibby Sterilin) in DMEM, 10\% FBS, supplemented with $50 \mathrm{ng} / \mathrm{mL}$ insulin (Sigma), $50 \mathrm{ng} / \mathrm{mL}$ hydrocortisone (Sigma), $25 \mathrm{ng} / \mathrm{mL}$ basic Fibroblast Growth Factor (Sigma), $20 \mathrm{ng} / \mathrm{mL}$ Epidermal Growth Factor (Sigma), B27 Supplement (Gibco) and $4 \mathrm{ug} / \mathrm{mL}$ heparin (BMS, Bristol Myers Squibb). Mammospheres were harvested once a week, using $0.05 \%$ trypsin $-0.53 \mathrm{mM}$ EDTA. The cell suspension was microscopically analyzed to check for the presence of spheres. Generated spheres were trypsinized again, to have a single cell suspension.

\section{Mammosphere differentiation}

Spheres generated from single LA7 cells were harvested and plated onto home-made collagen 
coated dishes and growth medium. Half of the medium was changed every 2-3 days. After 2 weeks, the differentiated outgrowths were fixed with PBS containing 4\% paraformaldehyde (PFA, Sigma).

\section{Collagen preparation}

For embedding in collagen gels, LA7 were resuspended on ice in a collagen solution prepared as described previously (Soriano et al. 1995). Cells were suspended at a density of 1000 cells $/ \mathrm{mL}$ and $1-\mathrm{mL}$ aliquots were dispensed into 24-mm wells (Nunc, Kampstrup, Roskilde, Denmark). After incubation at $37^{\circ} \mathrm{C}$ for at least $10 \mathrm{~min}$ to allow collagen gel formation, the complete medium was added and changed every 2-3 days.
Matrigel preparation

LA7 cells were embedded in Growth Factor Reduced Matrigel (Becton Dickinson). Briefly, cells were suspended in cold Matrigel at a density of 1000 cells $/ \mathrm{mL}$ in 24 -well plates. About $500 \mu \mathrm{L}$ of complete medium was added after polymerization and half of medium was changed every 2-3 days.

Cell fixation, staining and confocal microscopy analysis

Collagen gel cultures were washed twice with PBS and fixed for 10 min with 4\% PFA. After extensive rinsing with PBS, the collagen embedded cells were stained for 10 min with Hoechst nuclear dye 33342 (Sigma). Confocal analysis was performed with a Leica confocal microscope.
Fig. 1 Morphological characterization of LA7 cells at early and late passage and culture conditions. Panels (a) and (b): LA7 cells grown as a monolayer in

undifferentiating conditions maintain the same morphology with early and late tissue culture passages: passage 13 (a) or passage 50 (b). Panels (c) and (d): LA7 single cell-derived mammospheres maintain the same morphology for over 45 passages: passage 8 (c) or passage 47 (d). Panel (e): LA7 cells grown as a monolayer (panel a) or in non-adherent conditions (panel c), do not express differentiation markers. Lane 1: rat mammary gland positive control; lane 2 : undifferentiated LA7 cells at early passage (passage 13) in monolayer condition; lane 3: undifferentiated LA7 cells at early passage (passage 3) in suspension condition. Magnifications: $10 \times$, panels (a) and (b); $20 \times$, panels (c) and (d)
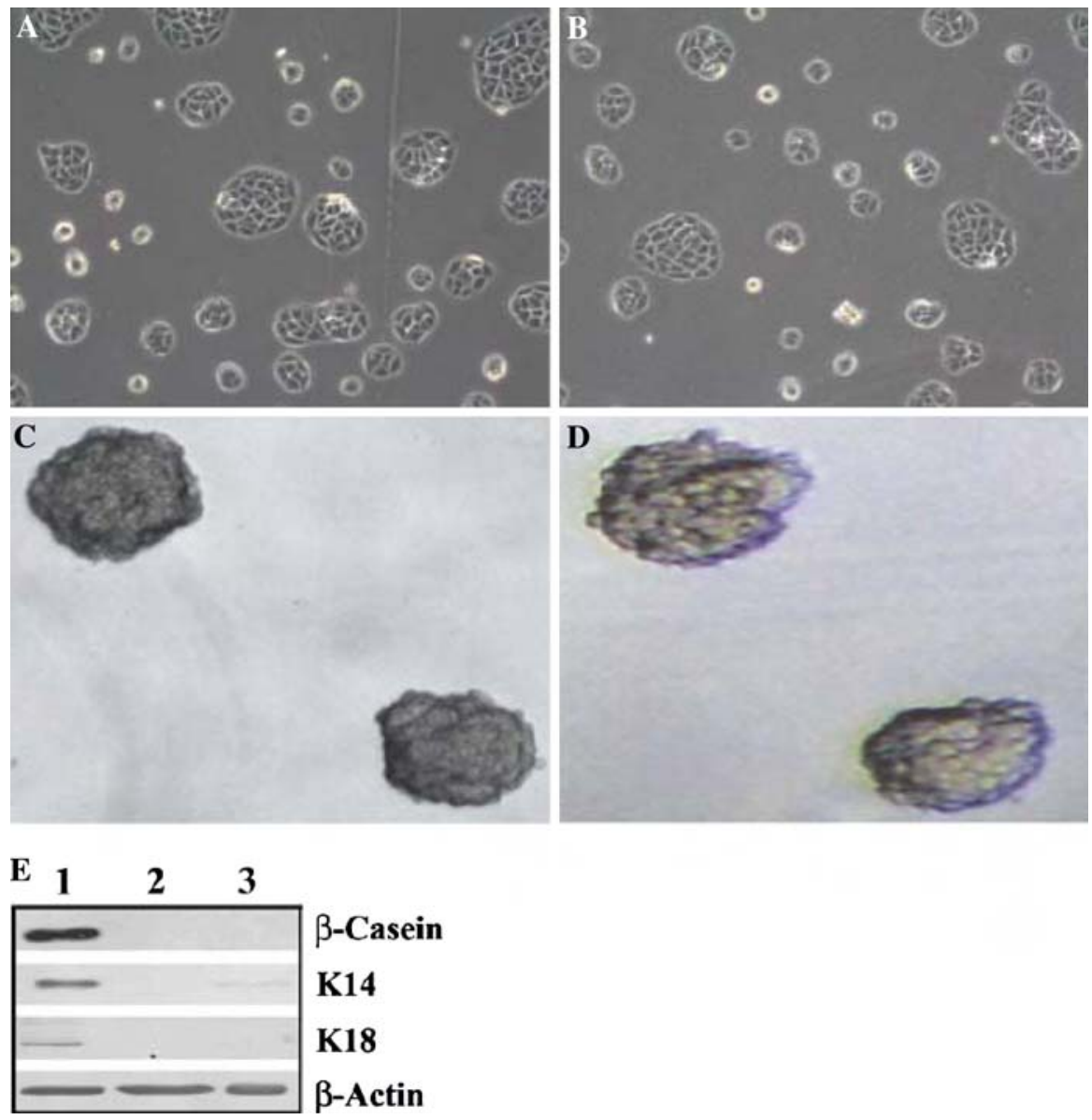
Western blot analysis

LA7 cells and rat mammary gland tissue, used as positive control, were lysed on ice using Laemmli buffer (100 mM TRIS pH 7, $200 \mathrm{mM}$ DTT, 20\% glycerol, $0.1 \%$ bromophenol blue, $4 \%$ SDS). About $50 \mu \mathrm{g}$ of total protein were loaded for each sample onto SDS denaturing poly-acrylamide gels. After transfer to PVDF membrane (GE-Biotechnology), the membrane was incubated with primary antibodies: monoclonal anti-cytokeratin 14 (Sigma), monoclonal anti-cytokeratin 18 (Becton Dickinson), and anti- $\beta$ casein (Santa Cruz). The following antibodies rabbit anti-goat, goat anti-mouse, and goat anti-rabbit were used as secondary antibodies (all purchased from Santa Cruz).

\section{Results}

LA7 cell line has self-renewal ability

Here, we demonstrate that LA7 cells, characterized by a typical epithelial polygonal morphology, can be
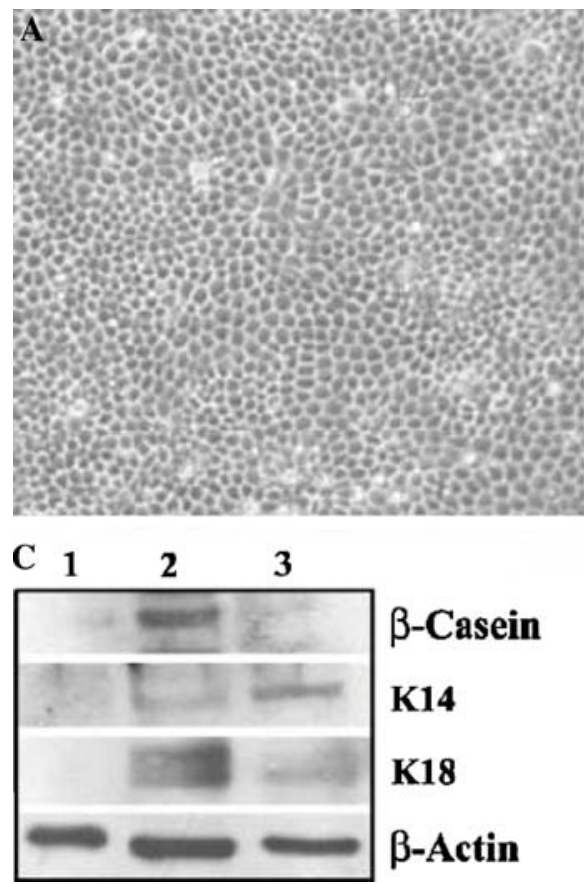

Fig. 2 LA7 cell differentiation and marker analysis. Panels (a) and (b): LA7 confluent cells (a); DMSO-induced differentiation result in dome-formation (b). Domes are hemispherical blisters formed by the detachment of the cell layer from the plastic dish by focal accumulation of liquid. Panel (c): Western propagated indefinitely (Fig. 1, panels a, b). Since recent evidence suggested that the suspension culture method allows for the in vitro propagation of stem cells (Reynolds et al. 1992), we used the mammosphere suspension culture approach to determine the in vitro self-renewing potential of LA7 cells. We determined the mammosphere formation efficiency, starting from single LA7 cells to be $85 \%$. As in the case of monolayer cultures, LA7-mammospheres could be regenerated indefinitely from cells derived from previously dissociated mammospheres (Fig. 1, panels $\mathrm{c}, \mathrm{d}$ ).

We then tested whether LA7 cells can maintain their undifferentiated status grown at monolayer subconfluent cultures or in non-adherent conditions. Moreover, we have analyzed by Western blot the expression of mammary gland cell lineage specific markers. We first determined that LA7 cells cultured as a monolayer and as mammospheres did not express the proteins cytokeratin 14 (marker for myoepithelial cells), cytokeratin 18 (marker for luminal cells) or $\beta$ casein (a marker for differentiated alveoli) (Fig. 1, panel e, lanes 2 and 3 respectively).

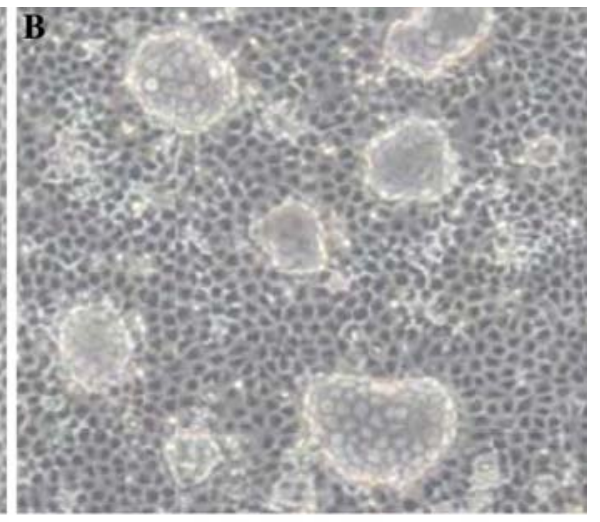

blot analysis of cells collected before and after DMSO induction and tested for mammary gland lineage specific differentiation markers. Lane 1: undifferentiated LA7 cells, lane 2: DMSO-differentiated LA7 cells, lane 3: rat mammary positive control. Magnifcation: $10 \times$, panels (a) and (b) 
LA7 cells have tri-lineage differentiation potential and the capacity to generate branched-like structures in $3 \mathrm{D}$ culture assays

The mammary gland is composed of stem/progenitor cells, and terminally differentiated luminal, alveolar and myoepithelial cells. We investigated the capacity of LA7 cells to terminally differentiate into mammary specific cell lineages. To this aim we treated LA7 confluent monolayer cultures (Fig. 2, panel a) with differentiation-inducers such as DMSO for $48 \mathrm{~h}$ and observed the appearance of domes, structures reminiscent of alveoli (Fig. 2, panel b). The expression of specific markers was assessed by Western blot analysis and it was determined that DMSO-differentiated LA7 cells express cytokeratin 14, cytokeratin 18 , and $\beta$-casein (Fig. 2, panel c, lane 2).

Mammosphere differentiation was performed by seeding a single LA7-generated mammosphere onto collagen-coated dishes (Fig. 3). One day after plating, an outgrowth from a LA7-mammosphere started to form (Fig. 3, panel b). At day 14 the mammosphere outgrowth revealed branched-like structures reminiscent of tubules (Fig. 3, panels c, d). The LA7mammosphere outgrowths were composed of myoepithelial and luminal cells as indicated by cytokeratin 14 and cytokeratin 18 expression (Fig. 3, panel e, lane 3). When treated with lactogenic hormones, such as prolactin, insulin and hydrocortisone, LA7-mammospheres started to produce milkassociated proteins such as $\beta$-casein. These differentiation assays demonstrate that LA7 cells have the differentiation potential to generate all of the cell lineages of the mammary gland and the ability to generate rudimentary branched structures.

\section{LA7 cells have the ability to generate 3D}

branched duct-like structures that recapitulate morphologically and functionally the ductalalveolar-like architecture of the mammary tree

We have shown that LA7 cells can be induced to form branched-like structures in $2 \mathrm{D}$ cultures. Consistent with this, a single LA7 cell seeded into a collagen gel, generated 3D tubule-like structures that are characterized with complex ramifications reminiscent of the mammary tree (Fig. 4, panel a). To determine whether LA7-generated tubule-like structures contained a lumen, we stained LA7 cell nuclei
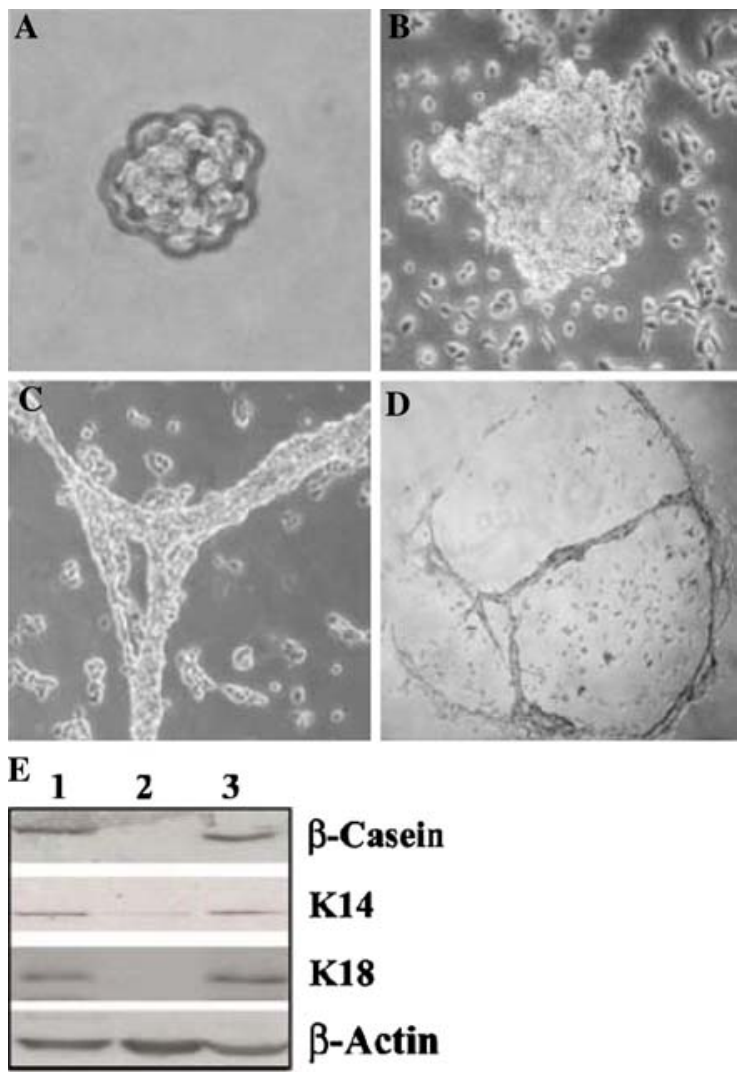

Fig. 3 Single LA7 cell-generated mammosphere and outgrowth analysis. Panel (a-d): a single mammosphere generated by a single LA7 cell plated onto collagen-coated dish (a); mammosphere-outgrowth at day 1 after plating (b); mammosphere-outgrowth two weeks after plating and tubular-like structures reminiscent of the mammary tree (c and d). Magnifications: $20 \times$, panels (a) and (b); $10 \times$, panel (c); $4 \times$, panel (d). Panel (e): Western Blot analysis on cells obtained from mammospheres. Differentiated mammosphere-derived outgrowths expressed lineage specific markers after two-week differentiation. Lane 1: rat mammary gland positive control; lane 2: cells derived from a mammosphere; lane 3: cells obtained from mammosphere-outgrowth at passage 3

with Hoechst 33342, and with confocal microscopy we found that the nuclear staining clearly outlined the lumen of the tubules (Fig. 4, panel b). Consistent with this observation, cyst-like and dome-like structures, obtained from LA7 cells, had the capacity for milk protein production (Fig. 4, panels c, d). Cysts and domes recapitulate certain aspects of alveologenesis in vitro (Zucchi et al. 2002). Cells obtained from these structures generated from a single LA7 cell were collected and also tested for the expression of cytokeratin 18 and 14 proteins (Fig. 4, panel d, 
Fig. 4 3D culture and marker analysis on structures generated by LA7 cells. Panels (a, b): LA7single cell generated tubules in collagen gel (a); tubules containing lumen, upper left part of the panel (b). Cells were stained with the Hoechst nuclear dye and analyzed with confocal microscopy (b). Panel (c): LA7-single cell generated cyst in Matrigel. Panel (d): cells collected from LA7derived tubules express luminal (K18), alveolar $(\beta$-casein) and myoepithelial (K14) markers. Lane 1: undifferentiated LA7 cells, lane 2: cells derived from a single LA7-generated tubules
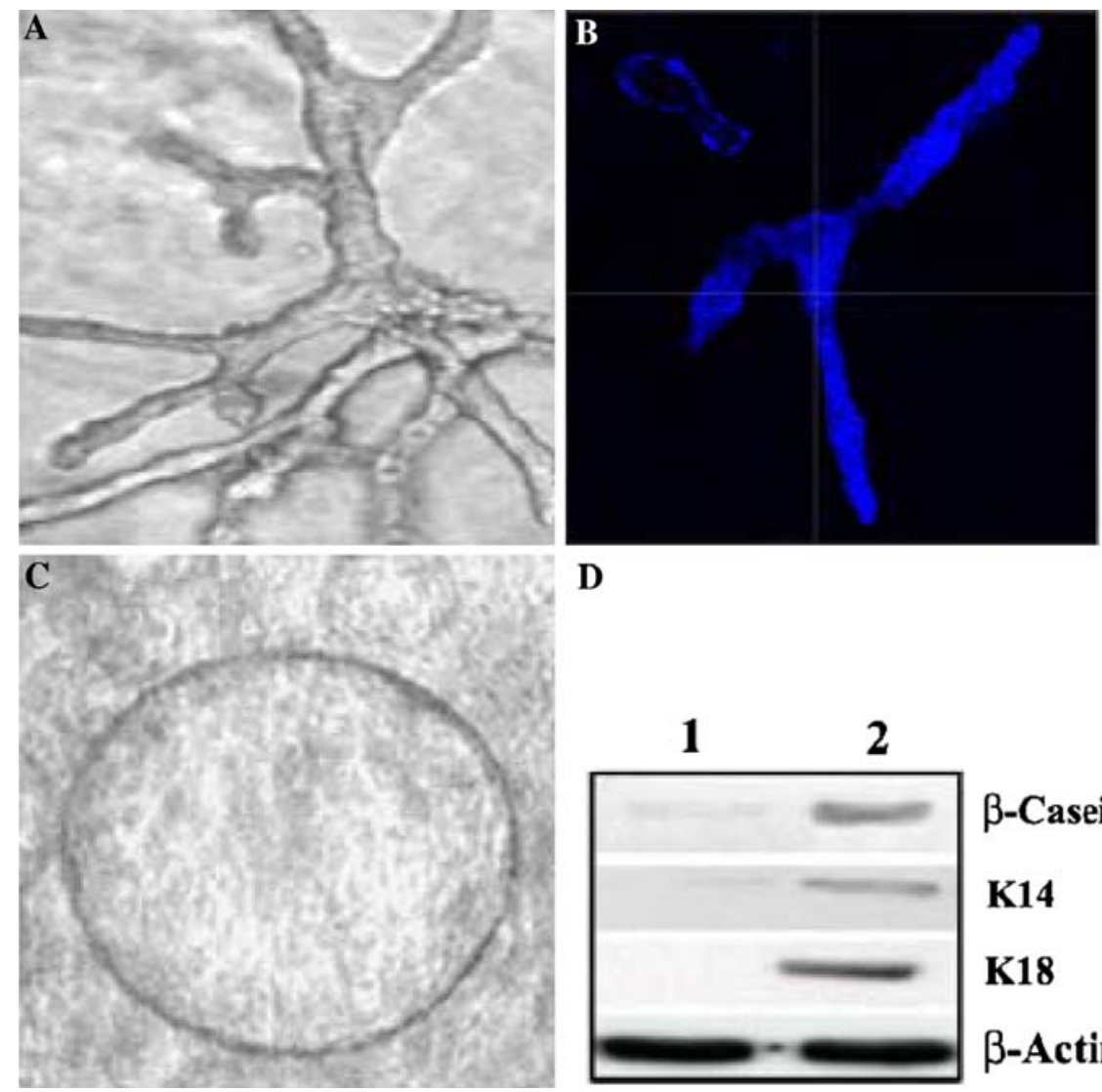

D

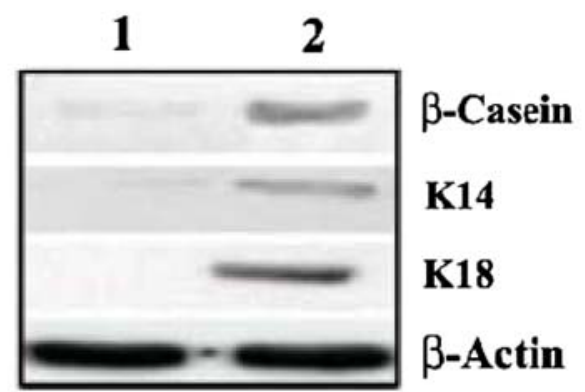

lane 2). These analyses show that LA7 cells are also a model for investigation of mammary gland differentiation in $3 \mathrm{D}$ cultures.

\section{Discussion}

Accumulating evidence supports the hypothesis that breast cancer might be a cancer stem cell disease. It has been reported that small subpopulations of human primary cells are able to sustain mammary tumor growth in vivo (Al-Hajj et al. 2003). However, a complete characterization of cancer stem cells is lacking due to the paucity of the stem cells in the mammary tissue, the scarcity of the available human tumor samples and the heterogeneity of the tissue complicating quantitative analysis. Most stem cell enrichment and purification protocols rely on fluorescence activated cell sorting (FACS) or magnetic immunosorting, using sets of antibodies against putative cell surface antigens specific for stem and/ or cancer stem cells. In the case of mammary gland stem cells, the lack of a specific expression signature makes cancer stem cell separation complex, problematic and often inconclusive.

Studies on the highly proliferative subventricular zone of rat embryos demonstrated that neural cells can be clonally propagated in suspension cultures, and can generate spherical colonies, termed neurospheres (Reynolds et al. 1992). This experimental system has proven to be an extremely useful tool in neural stem cell biology, for early development studies (Kukekov et al. 1997), to identify factors involved in cell fate determination and to unravel signaling pathways active in stem and early progenitor cells (Wafford et al. 2001; Zhu et al. 1999; Kawaguchi et al. 2001). The neurosphere culture is also routinely used now to assess for the enrichment of stem cells (Morshead et al. 1994; Uchida et al. 2000) the size of the stem cell population in neural tissue in experiments aiming to investigate the role of Bmi I (Molofsky et al. 2003) PTEN (Groszer et al. 2001) LIF and Notch (Grandbarbe et al. 2003; Shimazaki et al. 2001) in cell self-renewal. 
Mammosphere generation was first demonstrated by Dontu et al., where they showed that mammospheres are highly enriched for undifferentiated stem/ progenitor cells. The authors assessed the ability of single cells isolated from mammospheres to generate multi-lineage colonies when cultured in the presence of factors that promote their differentiation (Dontu et al. 2003). Furthermore, Dontu et al. demonstrated that mammospheres are able to generate colonies that contain all three lineages of the adult mammary gland: myoepithelial, luminal and alveolar cells. Mammospheres contain cells capable of clonally generating complex functional structures in Matrigel and when plated on collagen generated outgrowths with the morphological and cellular characteristics associated with human mammary ductal/alveolar structures.

Here we define a cell suspension culture protocol that allows for a continuous and contiguous propagation of clonally derived LA7 mammospheres. We demonstrated that mammospheres are tripotent, both after monolayer and three-dimensional cell induced differentiation. We also showed that when LA7 cells are embedded in collagen, a mammary gland likearchitecture with branching morphology and hollow ducts can be reconstructed in vitro. Cysts are instead generated when LA7 cells are embedded into Matrigel.

Here we provide a cell model that can be manipulated with relative ease in order to study tumor development generated by a single cancer stem cell. LA7 cells may also represent an efficacious tool for drug discovery and cytotoxic testing of therapeutic agents.

Acknowledgments We especially thank Loredana Ansalone for extraordinary secretarial support; Drs. L. Emionite and F. Delucchi from the IST-Genova (Italy) for tissue preparation. IZ Grant support: The Italy-USA Project Grant N.527 B-B7; FIRB Internazionali Grant RBIN04CBSM_000, MIUR-FIRB Grant RBIP0695BB_003; Telethon grant GG004247, Net2Drug grant N. 037590, and the N.O.B.E.L. Grant funded by Fondazione CARIPLO.

\section{References}

Al-Hajj M, Clarke MF (2004) Self-renewal and solid tumor stem cells. Oncogene 23:7274-7282

Al-Hajj M, Wicha MS, Benito-Hernandez A, Morrison SJ, Clarke MF (2003) Prospective identification of tumorigenic breast cancer cells. Proc Natl Acad Sci USA 100:3983-3988
Bennett DC, Peachey LA, Durbin H, Rudland PS (1978) A possible mammary stem cell line. Cell 15:283-298

Bonnet D, Dick JE (1997) Human acute myeloid leukemia is organized as a hierarchy that originates from a primitive hematopoietic cell. Nature Med 3:730-737

Dontu G, Abdallah WM, Foley JM, Jackson KW, Clarke MF, Kawamura MJ, Wicha MS (2003) In vitro propagation and transcriptional profiling of human mammary stem/ progenitor cells. Gene Dev 17:1253-1270

Dulbecco R, Bologna M, Unger M (1979) Differentiation of a rat mammary cell line in vitro. PNAS 76:1256-1260

George AA et al (2001) Detection of leukemic cells in the $\mathrm{CD} 34+\mathrm{CD} 38$ - bone marrow progenitor population in children with acute lymphoblastic leukemia. Blood 97:3925-3930

Going JJ, Abd El-Monem HM, Craft JA (2001) Clonal origin of human breast cancer. J Pathol 194:395-397

Grandbarbe L, Bouissac J, Rand M, Hrabé de Angelis M, Artavanis-Tsakonas S, Mohier E (2003) Delta-Notch signaling controls the generation of neurons/glia from neural stem cells in a stepwise process. Development 130:1391-1402

Groszer M, Erickson R, Scripture-Adams DD, Lesche R, Trumpp A, Zack JA et al (2001) Negative regulation of neural stem/progenitor cell proliferation by the Pten tumor suppressor gene in vivo. Science 294:2186-2189

Kawaguchi A, Miyata T, Sawamoto K, Takashita N, Murayama A, Akamatsu W, Ogawa M, Okabe M, Tano Y, Goldman SA, Okano H (2001) Nestin-EGFP transgenic mice: visualization of the self-renewal and multipotency of CNS stem cells. Mol Cell Neurosci 17:259-273

Kukekov VG, Laywell ED, Thomas LB, Steindler DA (1997) A nestin-negative precursor cell from the adult mouse brain gives rise to neurons and glia. Glia 21:399-407

Miyamoto T, Weissman IL, Akashi K (2000) AML1/ETOexpressing nonleukemic stem cells in acute myelogenous leukemia with 8;21 chromosomal translocation. Proc Natl Acad Sci USA 97:7521-7526

Molofsky AV, Pardal R, Iwashita T, Park IK, Clarke MF, Morrison SJ (2003) Bmi-1 dependence distinguishes neural stem cell self-renewal from progenitor proliferation. Nature 425:962-967

Morshead CM, Reynolds BA, Craig CG, McBurney MW, Staines WA, Morassutti D, Weiss S, van der Kooy D (1994) Neural stem cells in the adult mammalian forebrain: a relatively quiescent subpopulation of subependymal cells. Neuron 13:1071-1082

Ponti D, Costa A, Zaffaroni N, Pratesi G, Petrangolini G, Coradini D, Pilotti S, Pierotti MA, Daidone MG (2005) Isolation and in vitro propagation of tumorigenic breast cancer cells with stem/progenitor cell properties. Cancer Res 65:5506-5511

Reya T, Morrison SJ, Clarke MF, Weissman IL (2001) Stem cells, cancer, and cancer stem cells. Nature 414:105-111

Reynolds B, Tetzlaff W, Weiss S (1992) A multipotent EGF responsive striatal embryonic progenitor cell produces neurons and astrocytes. J Neurosci 12:4565-4574

Shackleton M, Vaillant F, Simpson KJ, Sting J, Smyth KG, Asselin-Labat ML, Wu L, Lindeman G, Visvader JE (2006) Generation of a functional mammary gland from a single cell. Nature 439:84-88 
Shimazaki T, Shingo T, Weiss S (2001) The ciliary neurotrophic factor/leukemia inhibitory factor/gp130 receptor complex operates in the maintenance of mammalian forebrain neural stem cells. J Neurosci 21:7642-7653

Soriano JV, Pepper MS, Nakamura T, Orci L, Montesano R (1995) Hepatocyte growth factor stimulates extensive development of branching duct-like structures by cloned mammary gland epithelial cells. J Cell Sci 108:413-430

Stingl J, Eirew P, Ricketson I, Shackleton M, Vaillant F, Choi D, Li HI, Eaves CJ (2006) Purification and unique properties of mammary epithelial stem cells. Nature 439:993-997

Uchida N, Buck DW, He D, Reitsma MJ, Masek M, Phan TV, Tsukamoto AS, Gage FH, Weissman IL (2000) Direct isolation of human central nervous system stem cells. Proc Natl Acad Sci 97:14720-14725

Wafford KA et al (2001) Growth factors regulate the survival and fate of cells derived from human neurospheres. Nat Biotechnol 19:475-479
Zhu G, Mehler MF, Mabie PC, Kessler JA (1999) Development changes in progenitor cell responsiveness to cytokines. J Neurosci Res 56:131-145

Zucchi I, Bini L, Albani D, Valaperta R, Liberatori S, Raggiaschi R, Montagna C, Susani L, Barbieri O, Pallini V, Vezzosi P, Dulbecco R (2002) Dome formation in cell cultures as expression of an early stage of lactogenic differentiation of the mammari gland. Proc Natl Acad Sci USA 99:8660-8665

Zucchi I, Sanzone S, Astigiano S, Pelucchi P, Scotti M, Valsecchi V, Barbieri O, Bertoli G, Albertini A, Reinbold RA, Dulbecco R (2007) The properties of a mammary gland cancer stem cell. Proc Natl Acad Sci USA 104:10476-10481 